\title{
Field Efficacy of Certain Chemicals and Neem Products against Shoot and Fruit Borer (Leucinodes orbonalis Guenee) on Brinjal (Solanum melongena L.) in Trans Yamuna Region of Allahabad
}

\author{
P.S. Kolhe*, Ashwani Kumar and A.R. Tayde \\ Department of Entomology, Sam Higginbottom University of Agriculture Technology and \\ Sciences, Allahabad-211007, India \\ *Corresponding author
}

\begin{tabular}{|c|c|}
\hline & A B S T R A C T \\
\hline Keywords & \multirow{6}{*}{$\begin{array}{l}\text { Field experiments were conducted during kharif2016-17 at central agriculture } \\
\text { field, SHUATS (Sam Higginbottom University of Agriculture, Technology and } \\
\text { Sciences), Allahabad, Uttar Pradesh (India). To determine field efficacy of certain } \\
\text { chemicals and neem products against shoot and fruit borer (Leucinodes orbonalis } \\
\text { Guenee) on brinjal (Solanum melongena L.) in Trans Yamuna region of } \\
\text { Allahabad. The result showed that the carbosulfan } 25 \text { EC @ } 0.05 \% \text { was found } \\
\text { most effective and showed }(6.50 \%) \text { shoot infestation, per cent fruit infestation } \\
(8.70 \%) \text { followed by Quinalphos } 20 \text { EC @ 0.05\% (7.96\%) and }(9.75 \%) \text {, } \\
\text { Cypermethrin } 25 \text { EC @ } 0.05(8.02 \%) \text { and (10.29), Chlorpyriphos 20EC @ } 0.05 \% \\
(8.07 \%) \text { and ( } 11.42 \%) \text {, Neem oil @ } 2 \%(8.61 \%) \text { and }(11.62 \%) \text {, NSKE @ 5\% } \\
(10.94 \%) \text { and }(12.58 \%) \text { and Neem leaf extract @ } 5 \%(12.11 \%) \text { and }(16.45 \%) \\
\text { respectively. }\end{array}$} \\
\hline & \\
\hline & \\
\hline $\begin{array}{l}\text { Insecticides, Neem } \\
\text { products. }\end{array}$ & \\
\hline Article Info & \\
\hline $\begin{array}{l}\text { Accepted: } \\
\text { 17 July } 2017 \\
\text { Available Online: } \\
\text { 10 September } 2017\end{array}$ & \\
\hline
\end{tabular}

\section{Introduction}

Vegetable cultivation is one of the most profitable and dynamic branches of agriculture. It has become an important source of income for both farmers and field labours, serving as a vehicle for reducing poverty in rural areas. Brinjal (Solanum melongena Linnaeus) also known as eggplant is referred as the "King of vegetables" originated from India and now grown as a vegetable throughout the tropical, sub-tropical and warm temperate areas of the world. It is a most important vegetable in the Indian Subcontinent that accounts for almost $50 \%$ of the world's area under its cultivation area under its cultivation. However, in India, the area is estimated as $7.5 \%$ of the total area of vegetables with $8 \%$ of the total production of vegetables (Roy et al., 2016). Brinjal (Solanum melongena L.) is one of the major vegetables in India extensively grown under diverseagro-climatic conditions throughout the year. Due to its nutritive value, consisting of minerals like iron, phosphorous, calcium and vitamins like $\mathrm{A}, \mathrm{B}$ and $\mathrm{C}$, unripe fruits are used primarily as vegetable in the country (Singh et al., 2016). Brinjal is one of the most important solanaceous vegetables in SouthEast Asian countries. The major brinjal 
growing states in India are Andhra Pradesh, Karnataka, West Bengal, Tamil Nadu, Maharashtra, Orissa, Uttar Pradesh, Bihar and Rajasthan. Globally, India ranks second and China ranks first in the production of brinjal (57.9\% of world output). In India, this crop occupies 71.13 lakh hectare area along with annual production of 135.57 (lakh tone) and productivity 19.1 MT per hectare. In Uttar Pradesh, the area under cultivation of brinjal is 3430 hectare producing 111.70 MT and the productivity is $8 \mathrm{MT} / \mathrm{ha}$. Leucinodes orbonalis (Guen.). The major constraints in economic cultivation of brinjal, insect pest infestation is a major bottleneck which poses threat for its production reported that the shoot and fruit borer (on shoot) were more prevalent during vegetative phase of crop. The yield loss by this pest varied from 0.08 $1.11 \mathrm{q} / \mathrm{ha}$ based on inconsumable pest of damaged fruits and 0.46- $3.80 \mathrm{q} / \mathrm{ha}$ when whole of the damaged fruits was taken into consideration. The borer infestation was $78.66 \%$ on top shoots in vegetative phase and then shifted to flowers and fruits with infestation reaching $66.66 \%$ in fruiting phase (Yadav et al., 2015). Leucinodes orbonalis (Guen.) (Lepidoptera: Pyralidae) is an internal borer which damages the tender shoots and fruits. Attack of this pest causes considerable damage to brinjal crop each year, affecting the quality and yield of the crop. The Larvae of this pest cause $12-16 \%$ damage to shoots and $2060 \%$ damage to fruits. The pest is very active during rainy and summer season and often causes more than up to $95 \%$ in India. It is also reported that the infestation of fruit borer causes reduction in Vitamin $\mathrm{C}$ content to an extent of $68 \%$ in the infested fruits (Anwar et al., 2015).

The management of this pest is through calendar spraying of conventional insecticides irrespective of pest incidence. Insecticides such as bio-pesticides, botanicals and chitin synthesis inhibitors, have been evaluated against (L. orbonalis) in the pest (Chatterjee and Roy, 2004) and are being used, besides the conventional insecticides. The increased dependence on pesticides, calendar based sprays by the farmer and/or short residual action of certain group of insecticides have not only lead to higher costs of production but also have not resulted in adequate control of pest. The extensive and indiscriminate use of pesticides for fruit and shoot borer management has led to several problems like resurgence of secondary pests, health hazards and pesticide residues inedible. Hence, present investigation was undertaken to evaluate the performance of certain chemicals and Neem products at their recommended dosages against fruit and shoot borer ( $L$. orbonalis) of brinjal (Kabir et al., 1996).

\section{Materials and Methods}

The present investigation was undertaken to evaluate efficacy of certain chemicals and neem products against shoot and fruit borer on brinjal at the Central Research Farm of Sam Higginbottom University of Agriculture, Technology and Sciences, Allahabad, Uttar Pradesh during Kharif season 2016. The research farm is situated on the right side of Allahabad.

Field trial was laid out in randomized block design (RBD) with 3 replications and 8 treatments including untreated control during kharif 2016-17 to evaluate the efficacy of four chemicals i.e., carbosulfan $0.05 \%$, quinalphos $0.05 \%$, cypermethrin $0.05 \%$, Chlorpyriphos $0.05 \%$ and with compared neem oil $2 \%$, NSKE 5\%, neem leaf extract 5\% against shoot and fruit borer on brinjal. Crop was raised in plots measuring $2 \times 2 \mathrm{~m}$ with a spacing $60 \times 60 \mathrm{~cm}$ between rows and plant, respectively. Transplanting was done on July $29^{\text {th }}$ in 2016.Crop was raised according to all agronomic package of practices under irrigated condition except the plant protection 
measure. Three rounds of insecticidal spray of different treatment were imposed on need basis during the crop season.

All the treatments were imposed by using hand compression sprayer. First spray was given 42 days after transplanting $\left(10^{\text {th }}\right.$ Sept 2016) and the remaining sprays was given at fortnightly intervals. The spraying was done during evening hours and care was taken to avoid drift of insecticides. Nosprays were given in untreated control.

\section{Data collection}

Five plants were randomly selected from each plot and tagged. Periodic observations were $3^{\text {rd }}, 7$ th, 14 th days after spray throughout the cropping season.

\section{On shoot}

\section{Number basis}

The total number of shoots and number of shoots infested of five selected plants from each treatment replication wise were recorded.

$\%$ Shoot infestation $=\frac{\text { No. of shoot infested }}{- \text { Total no. of shoot }}$

\section{On fruit}

\section{Number basis}

At each picking, the total number of fruits and number of fruits infested of five selected plants from each treatment replication wise were recorded.

$\%$ Fruit infestation $=\frac{\text { No. of fruit infested }}{\text { Total no. of fruit }}$

(Yadav et al., 2015)

\section{Statistical analysis}

Data were analyzed by using MSTAT software for analysis of variance. Percentage of shoot and fruit damaged by ESFB was transformed before analysis.

ANOVA was made by $F$ variance test and the pair comparisons were performed by Duncan's Multiple Range Test (Gomez and Gomez 1984).

\section{Results and Discussion}

\section{First spray- per cent shoot infestation}

The data on the mean $\left(3^{\text {rd }}, 7\right.$ th, 14 th DAS $)$ Per cent infestation of shoot borer on first spray revealed that all the chemical treatments were significantly superior over control. Among all the treatments lowest Per cent shoot infestation was recorded in carbosulfan $(6.50 \%)$ followed by quinalphos $(7.96 \%)$ which is statistically par with the treatments cypermethrin $(8.01 \%)$ and Chlorpyriphos $(8.06 \%)$, and is followed by neem oil (8.60\%). Treatments NSKE (10.94\%) which is statistically at par with followed by treatment neem leaf extract $(12.10 \%)$ it is found be least effective but significantly superior over the control (Table 1).

\section{Second spray- per cent fruit infestation}

The data on the mean $\left(3^{\text {rd }}, 7\right.$ th, and $14^{\text {th }}$, DAS $)$ Per cent infestation of fruit borer on second spray revealed that all the chemicals and neem products treatments were significantly superior over control (Table 2).

Among all the treatments, the lowest Per cent fruit infestation was recorded in carbosulfan $(8.58 \%)$ followed by quinalphos $(10.04 \%)$ which is at par and followed by cypermethrin (10.86\%), Neem oil (11.52\%), Chlorpyriphos (11.66) and NSKE (11.72) are statistically at 
par with each other, followed by neem leaf extract $(15.27 \%)$ is found be least effective but significantly superior over the control.

\section{Third spray: per cent fruit infestation}

The data on the mean $\left(3^{\text {rd }}, 7\right.$ th, and $14^{\text {th }}$ DAS $)$ Per cent infestation of fruit borer on third spray revealed that all the chemical and neem product treatments were significantly superior over control. Among all the treatments, the lowest Per cent fruit infestation was recorded in carbosulfan $(8.80 \%)$ statistically at par with quinalphos $(9.44 \%)$. The treatments Cypermethrine $(9.72 \%)$, and Chlorpyriphos $(11.17 \%)$ followed by neem oil $(11.72 \%)$ which is at par with each other. Treatment followed next is NSKE (13.44\%) which is followed by neem leaf extract $(17.61 \%)$ is found be least effective but significantly superior over the control (Table 3).

Table.1 Efficacy of certain chemicals and neem products against shoot and fruit borer Leucinodes orbonalis on brinjal (First Spray): (Per cent shoot infestation)

\begin{tabular}{|c|c|c|c|c|c|c|}
\hline \multirow{3}{*}{\multicolumn{2}{|c|}{ Treatments }} & \multicolumn{5}{|c|}{ Per cent shoot infestation of Leucinodes orbonalis } \\
\hline & & \multirow{2}{*}{$\begin{array}{l}\text { One day } \\
\text { before } \\
\text { spray }\end{array}$} & \multicolumn{4}{|c|}{ After spray } \\
\hline & & & \multirow{2}{*}{\begin{tabular}{|l}
$3^{\text {rd }}$ Day \\
14.66 \\
$(22.45)$
\end{tabular}} & \multirow{2}{*}{$\begin{array}{l}\mathbf{7}^{\text {th }} \text { Day } \\
17.93 \\
(24.97)\end{array}$} & \multirow{2}{*}{$\begin{array}{l}\mathbf{1 4}^{\text {th }} \text { Day } \\
22.33 \\
(28.19)\end{array}$} & \multirow{2}{*}{$\begin{array}{l}\text { Mean } \\
18.31 \\
(25.25)\end{array}$} \\
\hline $\mathbf{T}_{\mathbf{0}}$ & Control & $\begin{array}{l}13.90 \\
(21.89)\end{array}$ & & & & \\
\hline $\mathbf{T}_{1}$ & Cypermethrin 25 EC & $\begin{array}{l}10.33 \\
(18.75)\end{array}$ & $\begin{array}{l}4.96 \\
(12.84)\end{array}$ & $\begin{array}{l}8.76 \\
(17.20)\end{array}$ & $\begin{array}{l}10.33 \\
(18.67)\end{array}$ & $\begin{array}{l}8.02 \\
(16.27)\end{array}$ \\
\hline $\mathbf{T}_{2}$ & Quinalphos 20 EC & $\begin{array}{l}9.33 \\
(17.79)\end{array}$ & $\begin{array}{l}4.23 \\
(11.86)\end{array}$ & $\begin{array}{l}8.33 \\
(16.77)\end{array}$ & $\begin{array}{l}11.33 \\
(19.66)\end{array}$ & $\begin{array}{l}7.96 \\
(16.10)\end{array}$ \\
\hline $\mathbf{T}_{\mathbf{3}}$ & Carbosulfan 25 EC & $\begin{array}{l}8.90 \\
(17.36)\end{array}$ & $\begin{array}{l}4.00 \\
(11.53)\end{array}$ & $\begin{array}{l}7.50 \\
(15.89)\end{array}$ & $\begin{array}{l}8.00 \\
(16.42)\end{array}$ & $\begin{array}{l}6.50 \\
(14.62)\end{array}$ \\
\hline $\mathbf{T}_{4}$ & Neem oil $2 \%$ & $\begin{array}{l}8.66 \\
(17.11)\end{array}$ & $\begin{array}{l}6.83 \\
(15.14)\end{array}$ & $\begin{array}{l}7.66 \\
(16.06)\end{array}$ & $\begin{array}{l}11.33 \\
(19.66)\end{array}$ & $\begin{array}{l}8.61 \\
(16.96)\end{array}$ \\
\hline $\mathbf{T}_{\mathbf{5}}$ & Neem Leaf Extract $5 \%$ & $\begin{array}{l}11.50 \\
(19.82)\end{array}$ & $\begin{array}{l}10.33 \\
(18.74)\end{array}$ & $\begin{array}{l}11.83 \\
(20.11)\end{array}$ & $\begin{array}{l}14.16 \\
(22.10)\end{array}$ & $\begin{array}{l}12.11 \\
(20.32)\end{array}$ \\
\hline $\mathbf{T}_{6}$ & NSKE $5 \%$ & $\begin{array}{l}10.50 \\
(18.91)\end{array}$ & $\begin{array}{l}9.33 \\
(17.78)\end{array}$ & $\begin{array}{l}10.00 \\
(18.43)\end{array}$ & $\begin{array}{l}13.50 \\
(21.55)\end{array}$ & $\begin{array}{l}10.94 \\
(19.25)\end{array}$ \\
\hline $\mathbf{T}_{7}$ & Chlorpyriphos 20 EC & $\begin{array}{l}10.33 \\
(18.75)\end{array}$ & $\begin{array}{l}5.50 \\
(13.56)\end{array}$ & $\begin{array}{l}7.50 \\
(15.89)\end{array}$ & $\begin{array}{l}11.20 \\
(19.55)\end{array}$ & $\begin{array}{l}8.07 \\
(16.33)\end{array}$ \\
\hline \multicolumn{2}{|c|}{ Overall Mean } & 10.43 & 7.48 & 9.94 & 12.77 & 10.06 \\
\hline \multicolumn{2}{|c|}{ F- test } & NS & $\mathrm{S}$ & $\mathrm{S}$ & $\mathrm{S}$ & $\mathrm{S}$ \\
\hline \multicolumn{2}{|c|}{ S. Ed. $( \pm)$} & 3.719 & 0.808 & 1.088 & 0.671 & 0.794 \\
\hline \multicolumn{2}{|c|}{ C. D. $(P=0.05)$} & 7.884 & 1.714 & 2.307 & 1.423 & 1.682 \\
\hline
\end{tabular}

*Figures in parenthesis are arc sin transformed values. 
Table.2 Efficacy of certain chemicals and neem products against shoot and fruit borer Leucinodes orbonalis on brinjal ( $2^{\text {nd }}$ Spray Per cent fruit infestation)

\begin{tabular}{|c|c|c|c|c|c|c|}
\hline \multirow{3}{*}{\multicolumn{2}{|c|}{ Treatments }} & \multicolumn{5}{|c|}{ Per cent fruit infestation of Leucinodes orbonalis } \\
\hline & & \multirow{3}{*}{$\begin{array}{l}\begin{array}{l}\text { One day } \\
\text { before spray }\end{array} \\
11.33 \\
(19.67)\end{array}$} & \multicolumn{4}{|c|}{ After spray } \\
\hline & & & \multirow{2}{*}{$\begin{array}{l}\mathbf{3}^{\text {rd }} \text { Day } \\
14.00 \\
(21.88)\end{array}$} & \multirow{2}{*}{$\begin{array}{l}{7^{\text {th }} \text { Day }}^{17.50} \\
(24.67)\end{array}$} & \multirow{2}{*}{$\begin{array}{l}\mathbf{1 4}^{\text {th }} \text { Day } \\
24.00 \\
(29.33)\end{array}$} & \multirow{2}{*}{$\begin{array}{l}\text { Mean } \\
18.50 \\
(25.34)\end{array}$} \\
\hline $\mathbf{T}_{\mathbf{0}}$ & Control & & & & & \\
\hline $\mathbf{T}_{1}$ & Cypermethrine 25 EC & $\begin{array}{l}11.33 \\
(19.67)\end{array}$ & $\begin{array}{l}6.10 \\
(14.27)\end{array}$ & $\begin{array}{l}11.33 \\
(19.66)\end{array}$ & $\begin{array}{l}15.15 \\
(22.78)\end{array}$ & $\begin{array}{l}10.86 \\
(18.95)\end{array}$ \\
\hline $\mathbf{T}_{2}$ & Quinalphos 20 EC & $\begin{array}{l}12.00 \\
(20.27)\end{array}$ & $\begin{array}{l}4.93 \\
(12.82)\end{array}$ & $\begin{array}{l}11.05 \\
(19.41)\end{array}$ & $\begin{array}{l}14.16 \\
(22.10)\end{array}$ & $\begin{array}{l}10.05 \\
(18.11)\end{array}$ \\
\hline $\mathbf{T}_{3}$ & Carbosulfan 25 EC & $\begin{array}{l}11.05 \\
(19.42)\end{array}$ & $\begin{array}{l}3.76 \\
(11.18)\end{array}$ & $\begin{array}{l}9.83 \\
(18.27)\end{array}$ & $\begin{array}{l}12.16 \\
(20.40)\end{array}$ & $\begin{array}{l}8.58 \\
(16.62)\end{array}$ \\
\hline $\mathbf{T}_{4}$ & Neem oil $2 \%$ & $\begin{array}{l}11.66 \\
(19.97)\end{array}$ & $\begin{array}{l}6.56 \\
(14.84)\end{array}$ & $\begin{array}{l}11.50 \\
(19.82)\end{array}$ & $\begin{array}{l}16.50 \\
(23.96)\end{array}$ & $\begin{array}{l}11.52 \\
(19.54\end{array}$ \\
\hline $\mathbf{T}_{5}$ & Neem Leaf Extract $5 \%$ & $\begin{array}{l}11.50 \\
(19.82)\end{array}$ & $\begin{array}{l}10.33 \\
(18.74)\end{array}$ & $\begin{array}{l}15.50 \\
(23.18)\end{array}$ & $\begin{array}{l}20.00 \\
(26.56)\end{array}$ & $\begin{array}{l}15.28 \\
(22.83)\end{array}$ \\
\hline $\mathbf{T}_{6}$ & NSKE $5 \%$ & $\begin{array}{l}10.50 \\
(18.91)\end{array}$ & $\begin{array}{l}7.50 \\
(15.89)\end{array}$ & $\begin{array}{l}11.66 \\
(19.96)\end{array}$ & $\begin{array}{l}16.00 \\
(23.57)\end{array}$ & $\begin{array}{l}11.72 \\
(19.81)\end{array}$ \\
\hline $\mathbf{T}_{7}$ & Chlorpyriphos 20 EC & $\begin{array}{l}11.33 \\
(19.67)\end{array}$ & $\begin{array}{l}6.50 \\
(14.77)\end{array}$ & $\begin{array}{l}12.16 \\
(20.40)\end{array}$ & $\begin{array}{l}16.33 \\
(23.83)\end{array}$ & $\begin{array}{l}11.66 \\
(19.97)\end{array}$ \\
\hline \multicolumn{2}{|c|}{ Overall Mean } & 11.34 & 7.46 & 12.57 & 16.79 & 12.27 \\
\hline \multicolumn{2}{|c|}{ F- test } & NS & $\mathrm{S}$ & $\mathrm{S}$ & $\mathrm{S}$ & $\mathrm{S}$ \\
\hline \multicolumn{2}{|c|}{ S. Ed. $( \pm)$} & 3.093 & 0.920 & 0.892 & 1.173 & 0.556 \\
\hline \multicolumn{2}{|c|}{ C. D. $(P=0.05)$} & 6.557 & 1.950 & 1.890 & 2.487 & 1.179 \\
\hline
\end{tabular}

*Figures in parenthesis are arc sin transformed values.

Table.3 Efficacy of certain chemicals and neem products against shoot and fruit borer Leucinodes orbonalis on brinjal ( $3^{\text {rd }}$ Spray Per cent fruit infestation)

\begin{tabular}{|c|c|c|c|c|c|c|}
\hline \multirow{3}{*}{\multicolumn{2}{|c|}{ Treatments }} & \multicolumn{5}{|c|}{ Per cent Fruit infestation of Leucinodes orbonalis } \\
\hline & & \multirow{2}{*}{$\begin{array}{l}\text { One day } \\
\text { before } \\
\text { spray }\end{array}$} & \multicolumn{4}{|c|}{ After spray } \\
\hline & & & $3^{\text {rd }}$ Day & $7^{\text {th }}$ Day & $14^{\text {th }}$ Day & Mean \\
\hline $\mathbf{T}_{\mathbf{0}}$ & Control & $\begin{array}{l}24.66 \\
(29.77)\end{array}$ & $\begin{array}{l}26.33 \\
(30.85)\end{array}$ & $\begin{array}{l}28.50 \\
(32.25)\end{array}$ & $\begin{array}{l}32.83 \\
(34.94)\end{array}$ & $\begin{array}{l}29.22 \\
(32.69)\end{array}$ \\
\hline $\mathbf{T}_{1}$ & Cypermethrine 25 EC & $\begin{array}{l}17.33 \\
(24.60)\end{array}$ & $\begin{array}{l}7.16 \\
(15.50)\end{array}$ & $\begin{array}{l}9.50 \\
(17.94)\end{array}$ & $\begin{array}{l}12.50 \\
(20.70)\end{array}$ & $\begin{array}{l}9.72 \\
(18.05)\end{array}$ \\
\hline $\mathbf{T}_{2}$ & Quinalphos 20 EC & $\begin{array}{l}16.33 \\
(23.83)\end{array}$ & $\begin{array}{l}6.50 \\
(14.77)\end{array}$ & $\begin{array}{l}9.33 \\
(17.78)\end{array}$ & $\begin{array}{l}12.50 \\
(20.70)\end{array}$ & $\begin{array}{l}9.44 \\
(17.75)\end{array}$ \\
\hline $\mathbf{T}_{3}$ & Carbosulfan 25 EC & $\begin{array}{l}18.33 \\
(25.35)\end{array}$ & $\begin{array}{l}6.60 \\
(14.88)\end{array}$ & $\begin{array}{l}8.66 \\
(17.11)\end{array}$ & $\begin{array}{l}11.16 \\
(19.51)\end{array}$ & $\begin{array}{l}8.80 \\
(17.17)\end{array}$ \\
\hline $\mathbf{T}_{4}$ & Neem oil $2 \%$ & $\begin{array}{l}16.16 \\
(23.70)\end{array}$ & $\begin{array}{l}10.50 \\
(18.90)\end{array}$ & $\begin{array}{l}10.33 \\
(18.74)\end{array}$ & $\begin{array}{l}14.33 \\
(22.24)\end{array}$ & $\begin{array}{l}11.72 \\
(19.96)\end{array}$ \\
\hline $\mathbf{T}_{5}$ & Neem Leaf Extract $5 \%$ & $\begin{array}{l}20.50 \\
(26.92)\end{array}$ & $\begin{array}{l}14.50 \\
(22.38)\end{array}$ & $\begin{array}{l}16.50 \\
(23.96)\end{array}$ & $\begin{array}{l}21.83 \\
(27.85)\end{array}$ & $\begin{array}{l}17.61 \\
(24.73)\end{array}$ \\
\hline $\mathbf{T}_{6}$ & NSKE $5 \%$ & $\begin{array}{l}17.66 \\
(24.85)\end{array}$ & $\begin{array}{l}10.83 \\
(19.21)\end{array}$ & $\begin{array}{l}11.50 \\
(19.82)\end{array}$ & $\begin{array}{l}18.00 \\
(25.10)\end{array}$ & $\begin{array}{l}13.44 \\
(21.38)\end{array}$ \\
\hline $\mathbf{T}_{7}$ & Chlorpyriphos 20 EC & $\begin{array}{l}17.50 \\
(24.73)\end{array}$ & $\begin{array}{l}9.83 \\
(18.27)\end{array}$ & $\begin{array}{l}11.50 \\
(19.82)\end{array}$ & $\begin{array}{l}12.20 \\
(20.44)\end{array}$ & $\begin{array}{l}11.17 \\
(19.51)\end{array}$ \\
\hline \multicolumn{2}{|c|}{ Overall Mean } & 18.56 & 11.53 & 13.23 & 16.92 & 13.89 \\
\hline \multicolumn{2}{|c|}{ F- test } & NS & $\mathrm{S}$ & $\mathrm{S}$ & $\mathrm{S}$ & $\mathrm{S}$ \\
\hline \multicolumn{2}{|c|}{ S. Ed. $( \pm)$} & 3.381 & 0.794 & 0.739 & 0.850 & 0.888 \\
\hline \multicolumn{2}{|c|}{ C. D. $(P=0.05)$} & 7.168 & 1.684 & 1.567 & 1.802 & 1.882 \\
\hline
\end{tabular}

*Figures in parenthesis are arc sin transformed values. 
Table.4 Efficacy of certain chemicals and neem products against shoot and fruit borer Leucinodes orbonalis on brinjal ( $2^{\text {nd }}$ and $3^{\text {rd }}$ sprays over all mean $\%$ fruit infestation)

\begin{tabular}{|c|c|c|c|c|}
\hline \multicolumn{2}{|c|}{ Treatments } & \multirow{2}{*}{$\begin{array}{l}\mathbf{2}^{\text {nd }} \text { spray } \\
18.50 \\
(25.34)\end{array}$} & \multirow{2}{*}{$\begin{array}{l}\mathbf{3}^{\text {rd }} \text { spray } \\
29.22 \\
(32.69)\end{array}$} & \multirow{2}{*}{$\begin{array}{l}\text { Overall Mean } \\
23.86 \\
29.09)\end{array}$} \\
\hline $\mathbf{T}_{\mathbf{0}}$ & Control & & & \\
\hline $\mathbf{T}_{1}$ & Cypermethrine 25 EC & $\begin{array}{l}10.86 \\
(18.95)\end{array}$ & $\begin{array}{l}9.72 \\
(18.05)\end{array}$ & $\begin{array}{l}10.29 \\
(18.70)\end{array}$ \\
\hline $\mathbf{T}_{2}$ & Quinalphos 20 EC & $\begin{array}{l}10.05 \\
(18.11)\end{array}$ & $\begin{array}{l}9.44 \\
(17.75)\end{array}$ & $\begin{array}{l}9.75 \\
(18.18)\end{array}$ \\
\hline $\mathbf{T}_{3}$ & Carbosulfan 25 EC & $\begin{array}{l}8.58 \\
(16.62)\end{array}$ & $\begin{array}{l}8.81 \\
(17.17)\end{array}$ & $\begin{array}{l}8.70 \\
(17.14)\end{array}$ \\
\hline $\mathbf{T}_{4}$ & Neem oil $2 \%$ & $\begin{array}{l}11.52 \\
(19.54)\end{array}$ & $\begin{array}{l}11.72 \\
(19.96)\end{array}$ & $\begin{array}{l}11.62 \\
(19.93)\end{array}$ \\
\hline $\mathbf{T}_{5}$ & Neem leaf extract $5 \%$ & $\begin{array}{l}15.28 \\
(22.83)\end{array}$ & $\begin{array}{l}17.61 \\
(24.73)\end{array}$ & $\begin{array}{l}16.45 \\
(23.91)\end{array}$ \\
\hline $\mathbf{T}_{6}$ & NSKE $5 \%$ & $\begin{array}{l}11.72 \\
(19.81)\end{array}$ & $\begin{array}{l}13.44 \\
(21.38)\end{array}$ & $\begin{array}{l}12.58 \\
(20.76)\end{array}$ \\
\hline $\mathbf{T}_{7}$ & Chlorpyriphos 20 EC & $\begin{array}{l}11.66 \\
(19.67)\end{array}$ & $\begin{array}{l}11.18 \\
(19.51)\end{array}$ & $\begin{array}{l}11.42 \\
(19.75)\end{array}$ \\
\hline \multicolumn{2}{|c|}{ Overall Mean } & 12.27 & 13.89 & 13.08 \\
\hline \multicolumn{2}{|c|}{ F- test } & $\mathrm{S}$ & $\mathrm{S}$ & $\mathrm{S}$ \\
\hline \multicolumn{2}{|c|}{ S. Ed. $( \pm)$} & 0.556 & 0.888 & 2.729 \\
\hline \multicolumn{2}{|c|}{ C. D. $(P=0.05)$} & 1.179 & 1.882 & 5.786 \\
\hline
\end{tabular}

*Figures in parenthesis are arc sin transformed values.

Over all Mean $\left(2^{\text {nd }}\right.$ and $3^{\text {rd. }}$ sprays of fruit infestation)

The data on the overall mean $\left(2^{\text {nd }}\right.$ and $3^{\text {rd }}$ spray) Per cent infestation of fruit borer on spray revealed that all the chemical and neem product treatments were significantly superior over control. Among all the treatments, the lowest Per cent fruit infestation was recorded in carbosulfan $(8.70 \%)$ followed by quinalphos $(9.75 \%)$ and Cypermethrine $(10.29 \%)$, which are statistically at par with each other. Chlorpyriphos $(11.42 \%)$ which is statistically at par with neem oil (11.62\%) and NSKE (12.58\%) which is followed by neem leaf extract $(16.45 \%)$ is found be least effective but significantly superior over the control (Table 4).

The data on the mean $(3,7 \& 14$ DAS) Per cent shoot infestation of first spray revealed that all the chemical and neem product is effective and at par. Among all the treatments lowest Per cent infestation of shoot and fruit borer was recorded in Carbosulfan $25 \mathrm{EC}$ $(6.50 \%)$ these findings are in supported by Rahman et al., (2009) (7.59\% and 3.93\%), Roy et al., (2016) (4.33\%), Devi et al., (2014) (14.53\%), Quinalphos 20 EC (7.96\%) is found to the next best treatments which is in line with the findings of Tayde and Simon (2010) (12.63\%). Cypermethrin 25 EC $(8.02 \%)$ is found to the next best treatments which is in line with the findings of Yadav et al., (2015) (11.08\%), Kalawate and Dethe (2012) (13.10\%), Mainali et al., (2013) (4.27\%) Chlorpyriphos $(8.07 \%)$ is found to the next best treatments, Neem oil $(8.61 \%)$ was next effective treatment which was supported by Yadav et al., (2015) (11.22\%), Tayde and Simon (2010) (14.93\%), Singh et al., (2015) (5.66\%). Next to these treatments was NSKE (10.94\%)

The data on the Percent fruit infestation of shoot and fruit borer on mean of second and 
third spray revealed that all the treatments were significantly superior over control. Among all the treatments lowest Per cent infestation of fruit was recorded in Carbosulfan 25 EC (8.70\%) these findings are in support with Rahman et al., (2009) (4.38\% and 3.93\%), Latif et al., (2010) (6.80\%) Roy et al., (2016) (10.52\%), Devi et al., (2014) $(11.85 \%)$. Quinalphos $(9.75 \%)$ is found to the next best treatments, which is in line with the findings of Tayde and Simon (2010) (10.51\%). Cypermethrin (10.29\%) similar findings to this result is supported by Yadav et al., (2015) (10.41\%), Kalawate and Dethe (2012) (13.10\% and 7.70\%). Chlorpyriphos $(11.42 \%)$ is found to be the next effective treatments. These results are Similar with Yadav et al., (2015) (14.02\%), Neem oil $(11.62 \%)$ found to be next effective treatment, which is supported by Tayde and Simon (2010) (14.55\%), Yadav et al., (2015) (12.63\%), Kumar et al., (2017) (19.98\%). NSKE $(12.58 \%)$ was next treatment that was effective, this was similarly supported by Singh et al., (2016) (7.50\%), Karkar et al., (2014) (14.46\%)

\section{Acknowledgment}

The authors are thankful to Head, Department of Entomology, Sam Higginbottom University of Agriculture Technology and Sciences, Allahabad-211007 for providing the facilities during investigation.

\section{References}

Anwar, S., Mari, J.M.., Khanzada, M.A. and Ullah, F. 2015. Efficacy of insecticides against infestation of brinjal fruit borer, (Leucinodes orbonalis Guenee) (Pyralidae: Lepidoptera) under field conditions Journal of Entomology and Zoology Studies, 3(3): 292-295

Chatterjee, M.L., and Roy, S. 2004. Bioefficacy of some insecticides against brinjal shoot and fruit borer, Leucinodesorbonalis Guen. And effect of novaluron on natural enemies of brinjal pests. Pestol, 27(10): 52-56.

Devi, P., Sahu, T. K., Ahirwar, R. B. and Kostha, V. K. 2014. Field evaluation of insecticides for management of shoot and fruit borer, Leucinodes orbonalis Guenee in Brinjal International Quarterly Journal of Environmental Sciences, 6 (1): 463-466.

Gomez, K.A., and Gomez, A.A. 1984. Statistical procedure for agricultural research. An International Rice Research Institute Book. A Wileyinterscience Publication, Johan Wiley and Sons. New York. 680 P.

Kabir, K.H., M.E. Baksh, F.M.A. Rouf and A. Ahemad. 1996. Insecticides use pattern on vegetables at farmers' level of Jessore resign in Bangladesh. Bangladesh J. Agric. Res. 21(2): 214254.

Kalawate, A., and Dethe, M.D. 2012. Bio efficacy study of biorational insecticide on brinjal. J Biopest. 5(1):75-80.

Karkar, D.B., Korat, D.M. and Dabhi, M.R. 2014. Evaluation of botanicals for their bio-efficacy against insect pests of brinjal Karnataka J. Agric. Sci., 27 (2):145-147

Kumar, M., Shanti, K.S., Rajapandian, K., and Kavitha, P. 2017. Field Evaluation of Bio Formulation KKKP and Bio Pesticides against Brinjal Shoot and Fruit Borer, Leucinodes orbonalis Guenee under Semiarid Conditions of Tamil Nadu.Int. J. Pure App. Biosci. 5 (1): 840-843.

Latif, M.A., Rahman, M.M., Alam, M.Z. 2010. Efficacy of nine insecticides against shoot and fruit borer, Leucinodes orbonalis (Guenee) (Lepidoptera: Pyralidae) in eggplant. $J$ Pest Sci., 83: 391-397

Mainali, R.P., Thapa, R.B., Pokhrel, P., 
Dangi, N. and Aryal. S. 2013. Biorational management of eggplant fruit and shoot borer, Leucinodes orbonalis Guenee, (Lepidoptera: Pyralidae) in Lalitpur, Nepal, Journal of Plant Protection Society, 4: 235-247.

Rahman, M. M., Islam, K. S. Jahan, M. And Uddin, M. A. 2009. Efficacy of some botanicals in controlling brinjal shoot and fruit borer, Leucinodes orbonalis. Progress. Agric., 20(1-2): 35 - 42.

Roy, G., Gazmer, R., Sarkar S., Laskar, N., Das, G., and Samanta, A., 2016. Comparative bioefficacy of different insecticides against fruit and shoot borer (Leucinodes orbonalis Guenee) of Brinjal and their effect on natural enemies. International Journal of Green Pharmacy, 10(4): S257-S260

Singh, J.P., Gupta, P.K., Chandra, U., and V.K. Singh., 2016. Bioefficacy of newer insecticides and biopesticides against brinjal shoot and fruit borer Leucinodes orbonalis Guenee (Lepidoptera:
Pyralidae). International Journal of Plant Protection, Volume 9| Issue 1 | 17

Srinivasan, M. R. and Rabindra, R. J. 2001. Bioefficacy of new molecule against brinjal shoot and fruit borer. Proceeding of Second National Symposium on Integrated Pest Management (IPM) in Horticulture Crops. New Molecules, Biopesticides and Environment, 249250.

Tayde, A. R. and Simon, S. 2010. Efficacy of spinosad and neem products against shoot and fruit borer (Leucinodes orbonalis Guenee) of brinjal (Solanum melongena L.). Trendsin Biosc. 3 (2): 208-209.

Yadav, R., Lyall, H., Kumar, S., and Sanp, R.K. 2015. Efficacy of certain botanical insecticides against shoot and fruit borer (Leucinodes orbonalis Guenee), on brinjal (Solanum melongena L.), The Bioscan, 10(2): 987-990.

\section{How to cite this article:}

Kolhe, P.S., Ashwani Kumar and Tayde, A.R. 2017. Field Efficacy of Certain Chemicals and Neem Products against Shoot and Fruit Borer (Leucinodes orbonalis Guenee) on Brinjal (Solanum melongena L.) in Trans Yamuna Region of Allahabad. Int.J.Curr.Microbiol.App.Sci. 6(9): 1320-1327. doi: https://doi.org/10.20546/ijcmas.2017.609.159 